\title{
Statistical evaluation of the loosening of total hip replacement
}

\author{
H.F. Hildebrand \\ Groupe de Recherches sur les Biomatériaux, Faculté de Médecine, 1 place de Verdun, \\ 59045 Lille Cedex, France
}

This presentation is a call for partners participating in a European Implant Data Retrieval and Analysis System of Total Hip Replacements. The aim of the proposal is the development, i.e., hardware, software components and networks services, and the centralization of a European Implant Data Retrieval and Analysis System with special references to Total Hip Replacements. The system should be easily enlarged to other implant systems, i.e., continuously involve new partners and other member states which will become potential users of the EDRAS-THR Database.

This database will be fed by the results obtained from clinical and biological analyses, i.e.:

- Systematic studies of medical evaluation of THR needing an explantation;

- Rigorous assessment of generalized (immunological, inflammatory) and/or remote biological effects in patients with THR and showing complications;

- Systematic analyses of biological swaps taken during implant- or THR-retrieval

- to precise the structure of new-formed periprosthetic tissue;

- to identify eventual wear particles;

- to assess morphological elements allowing to understand the mechanism of a failure or a loosening of THR.

As to partner research, at least one complete team per member state should participate, including clinicians, analytical scientists, histologists, immunologists.

- orthopaedic clinicians and surgeons,

- histopathologists with special interest in bone tissue,

- cell biologists: transmission electron microscopy,

- analysts: energy dispersive spectrometry (EDS);

atomic absorption spectrometry (AAS);

ICP-MS,

- immunologists: inflammation mediators,

- data processing,

- industry producing THR.

It is a fundamental position of the EDRAS-THR team that multimedia information will be viewed as a set of multimedia and hypermedia information objects (MH-Objects). All information elements that form the common base of the envisioned Interactive Multimedia Database will be inserted and 
viewed through a coded representation of independent, elementary units which will be specified as MH-Objects. As such they will be handled and interchanged inside the proposed application.

The complete system will be validated on three criteria:

- Functional validation of individual components and subsystems will include testing for correctness and operability of individual system components;

- System validation will include testing and validation of interrelationships and functioning of all components set up together in the complete system;

- User validation will be concerned with user friendliness, operational efficiency, transparency, etc.

The demonstration of the system will be organized at all participant locations, i.e., at least one for each user in the Consortium. From that moment onward, the system will be established on a global basis. When satisfactorily completed, this globally integrated network will be gradually transferred to real-life usage, which will be in fact the main validation of the project.

After the project duration, a self-financement of the network is envisaged, which may be assured by financial participation of public organisations and/or by access fees for data output to be payed from general users not belonging to the initial EDRAS-THR Consortium.

The innovation of the present proposal for a EDRAS-THR Database is the assessment of clinical, biological and analytical data which clearly pushes the patient's interest in the foreground, i.e., the risk assessment is no longer restricted to the surgeon and implant but is enlarged to the patient, the real concerned person. The need of an European Database is emphasized by the widespread European distribution of most orthopaedic implants. 